\title{
LOW-ORDER MODELLING OF COMBUSTION NOISE IN AN AERO-ENGINE: THE EFFECT OF ENTROPY DISPERSION
}

\author{
Yasser Mahmoudi \\ Andrea Giusti ${ }^{\dagger}$ \\ Epaminondas Mastorakos \\ Ann P. Dowling \\ Department of Engineering \\ University of Cambridge \\ Trumpington Street, CB2 1PZ, Cambridge, UK \\ Email: ag813@cam.ac.uk
}

\begin{abstract}
The present work studies the effect of entropy dispersion on the level of combustion noise at the turbine outlet of the RollsRoyce ANTLE aero-engine. A new model for the decay of entropy waves, based on modelling dispersion effects, is developed and utilised in a low-order network model of the combustor (i.e. LOTAN code that solves the unsteady Euler equations). The proposed model for the dispersion of entropy waves only requires the mean velocity field as an input, obtained by RANS computations of the demonstrator combustor. LOTAN is then coupled with a low order model code (LINEARB) based on the semi-actuator disk model that studies propagation of combustion noise through turbine blades. Thus, by combining LOTAN and LINERAB the combustion noise and its counterparts, direct and indirect noise, generated at the turbine exit are predicted. In comparison with experimental data it is found that without the inclusion of entropy dispersion, the level of combustion noise at the turbine exit is over-predicted by almost two orders of magnitude. The introduction of entropy dispersion in LOTAN results in much better agreement with the experimental data, highlighting the importance of entropy wave dispersion for the prediction of combustion noise in real engines. In more detail, the agreement with the experiment for high and low frequencies was very good. At intermediate frequencies the experimental measurements are still over-predicted, however the predicted noise is much smaller compared to the case without entropy dispersion. This discrepancy is attributed to (i) the role of turbulent mixing in the overall decay of the entropy fluctuations inside the combustor, not considered in the model developed for the decay of entropy waves, and (ii) the absence of a proper model in LINEARB for the decay of entropy waves as they pass through the turbine blade rows. These are areas that still need further development to improve the prediction of low-order network codes.
\end{abstract}

\section{INTRODUCTION}

Combustion noise is a very important aspect to be considered in the design of aero-engines due to its impact on the overall noise emissions produced by an aircraft $[1,2]$. This is more prominent in the next generation combustion technologies where the high level of unsteadiness of the reacting process results in increased external noise from the combustion [3]. The total noise radiated by a gas turbine combustion chamber system consists of 'direct' and 'indirect' noise [4,5]. Pressure perturbations generated by fluctuation in heat release rate from the turbulent flame constitute the 'direct' acoustic noise. The 'indirect' noise is generated due to the acceleration of entropy and vorticity waves through the turbine blade rows [5-7]. Recent studies have shown that the indirect noise due to vorticity gives a negligible contribution to the total noise generated in the aircraft [3]. However, the level of indirect noise generated by the entropy is of the same order of magnitude or larger than the direct acoustic noise [3]. Therefore, in order to have a reliable prediction of the combustion noise in an aero-engine, it is important to properly incorporate the effect of all physical phenomena involved in the generation, propagation and dissipation of indirect entropy noise.

Entropy waves are known to disperse and diffuse during their convection (e.g. $[3,8,9])$, however these phenomena are usually not considered in low-order modelling tools for combustion noise prediction. The convection of entropy waves in low-order network

${ }^{*}$ Current Address: School of Mechanical and Aerospace Engineering, Queens University Belfast, Belfast, BT9 5AH, UK, email: s.mahmoudilarimi@ qub.ac.uk

${ }^{\dagger}$ Address all the correspondence to this author.

GTP-17-1244, A. Giusti 
codes is usually modelled in a very simple way with the entropy waves either advected without attenuation or completely diffused [10]. Attempts in modelling the entropy wave diffusion and dispersion in a more comprehensive way are represented by the theoretical work by Sattelmayer [11], where the dispersion of entropy waves due to a differential time delay is considered, and the more recent works by Morgans at al. [12] and Giusti et al. [13] where the convection of entropy spots has been analyzed through numerical simulations and transfer functions for the transport of entropy waves have been proposed. In particular, in Ref. [13], where the convection of entropy waves in a fully-developed pipe flow was investigated, the transport of entropy fluctuations was found to be affected by both the shear dispersion due to a non-uniform mean velocity profile, and diffusion and turbulent mixing. The presence of a non-uniform mean velocity profile causes a dispersion of the time delay (phase) of the entropy perturbations [11] which tends to reduce their cumulative effect $[3,11,12]$. In addition, diffusion and turbulent mixing further decrease the amplitude of the fluctuations. The effect of diffusion and turbulent mixing increases with the distance travelled by the wave and with the decrease of the wavelength of the entropy fluctuations. Because of such phenomena, the decay of entropy fluctuations is strongly dependent on the spatial location with the level of fluctuations that decreases faster close to the walls where, due to the small value of the convection velocity, the wavelength of the entropy fluctuations becomes smaller and smaller. Considering integral values over the cross section, one-dimensional transfer functions, useful for low-order network codes, can be developed [12,13]. As previously discussed, the decay of the entropy fluctuations is a function of both the distance travelled by the wave and the wavelength of the entropy fluctuation. It was found [13] that in a fully developed pipe flow the magnitude of the entropy transfer function (defined as the ratio, in the frequency domain, of the entropy component at a given location and the one at the inlet) scales well with a Helmholtz number defined as $H e=f z / U_{b}$, where $z$ is the axial distance along the duct and $U_{b} / f$ represents a reference wavelength for the entropy perturbation averaged over the cross section. A similar behaviour has also been recently observed through experiments in a model gas-turbine combustor [14], characterized by a flow field much closer to a real engine.

Mean velocity profiles obtained through RANS simulations can be exploited to get a more realistic evaluation of the entropy convection. The model proposed in Ref. [13], which considers only the effects of the differential time delay due to a nonuniform mean velocity profile, showed a good agreement with numerical simulations in terms of magnitude of the entropy transfer function at low values of $\mathrm{He}$ (i.e. short distances travelled by the wave and/or long wavelengths of the entropy perturbation), whereas for intermediate and high values of $\mathrm{He}$ some discrepancies were found, possibly due to the effect of diffusion and turbulent mixing not considered in the low-order model. On the contrary, a good agreement with numerical results in terms of phase of the entropy transfer function was found in the entire investigated range of $\mathrm{He}$, showing that the time delay of the entropy perturbation averaged over the cross section is mainly influenced by the mean velocity profile. This is a direct consequence of the fact that the transport of the entropy waves is based on the flow-field convection. It is important to point out that the effect of turbulent mixing might be more pronounced in real combustors where the presence of swirling flows and recirculation regions (typically used to stabilize the combustion) may have a strong influence on the decay of the perturbations. Furthermore, interaction of hot combustion products with fresh air coming from cooling and dilution ports may add additional contributions to the entropy fluctuations [15].

The main objective of this work is to investigate the effect of entropy dispersion on the prediction of the combustion noise at the exit of an aero-engine. To do that, the noise generation and propagation in the combustor and turbine of the Rolls-Royce ANTLE demonstrator engine, for which experimental measurements are available, have been analyzed. The investigation performed here is based on low-order network codes for both the combustor and the turbine [10,16-19]. In order to include the effects of entropy dispersion in the combustor, a new low-order model suitable for real combustor geometries is proposed. This model only requires the mean velocity field as an input which can be obtained through simple RANS simulations of the combustor.

The paper is organized as follows. First, the low-order networks used to model both the combustor and the turbine are presented together with the model proposed for the entropy dispersion. Then, results of simulations performed without and with entropy attenuation are discussed in order to highlight the effect of the entropy dispersion on the noise prediction. Conclusions and recommendations for future work close the paper.

\section{NUMERICAL METHODS}

The configuration investigated in this work is schematically shown in Fig. 1 and represents the combustor and turbine of the RollsRoyce ANTLE engine. The flow coming from the compressor enters an annular combustion chamber. Unsteady heat release rate leads to the generation of pressure and entropy waves which travel through the turbine, interacting with the nozzle vanes. The demonstrator engine combustor investigated in this work consists of a diffuser plenum and 16 premixer ducts which deliver fuel and air to the combustion chamber. The combustor is surrounded by inner and outer annuli which supply cooling air to the combustor through inner and outer perforated tiles. The demonstrator turbine comprises a number of HP, IP and LP stages and discharges through a jet pipe at the outlet. As detailed in the following, both the combustor and the turbine have been modelled using low-order network codes. In the engine demonstrator, measurements of the pressure level at the outlet, inside the combustor and in the outer annulus are available. Such 
measurements will be used in the following to assess the capability of low-order models to predict the combustion noise and analyze the effect of dispersion of entropy waves on the noise prediction.

\section{Combustor model}

The pressure perturbation $\widehat{p}(\mathbf{x}, \omega)$ generated by unsteady heat per unit volume $\widehat{q}(\mathbf{y}, \omega)$ in a combustor can be obtained by using the inhomogeneous wave equation in the frequency domain as:

$$
\widehat{p}(\mathbf{x}, \omega)=\int_{V_{f}} \widehat{G}_{1}(\mathbf{y}, \mathbf{x}, \omega) \widehat{q}(\mathbf{y}, \omega) \mathrm{d}^{3} \mathbf{y}
$$

where $\omega$ is the angular frequency, $V_{f}$ denotes the volume of the flame brush, $\widehat{G}_{1}$ is the Fourier transform of the Green's function that represents the pressure generated at $(\mathbf{x}, \omega)$ due to an impulse of rate of heat addition. This function is the pressure response of the linearised Euler equations to harmonic fluctuations of heat release rate that satisfies appropriate boundary conditions. In a similar way, entropy fluctuations can be obtained as a volume integral of the product of a different Green's function $\widehat{G}_{2}$ with the rate of heat release per unit volume. By using the Green's function technique, the pressure response to a concentrated source $\frac{1}{r} \delta\left(\theta-\theta_{0}\right) \delta\left(r-r_{0}\right) \delta(x-$ $\left.x_{0}\right) \exp (i \omega t)$ at circumferential angle $\theta_{0}$ can be expressed as a superposition of circumferential modes of the transfer function:

$$
\widehat{G}_{1}(\mathbf{y}, \mathbf{x}, \omega)=\sum_{n=-N}^{N}\left(\frac{\widehat{p}}{\widehat{q}}\right)_{n} \exp \left[\operatorname{in}\left(\theta-\theta_{0}\right)\right]
$$

where $(\widehat{p} / \widehat{q})_{n}$ is the modal transfer function. In the present work, the Green's functions $\widehat{G}_{1}$ and $\widehat{G}_{2}$ were computed using the low-order acoustic network code LOTAN $[10,16,17]$ (linearised Euler equations are solved and the entropy perturbation is obtained from pressure and density fluctuations, $\left.s^{\prime}=c_{v} p^{\prime} / \bar{p}-c_{p} \rho^{\prime} / \bar{\rho}\right)$. Once a model of the combustor is built, the LOTAN code directly calculates the modal transfer function and can hence be used to determine the frequency spectrum of the pressure at $(r, \theta, x)$ due to a source of unsteady heat input at $\left(r_{0}, \theta_{0}, x_{0}\right)$. By running LOTAN for different source positions and varying the values of $n$ the dependence of Green's function on the source position can be obtained [20]. It was found $[20,21]$ that the first 7 circumferential modes are sufficient to ensure accurate prediction of the Green's function for the geometry under investigation.

In LOTAN each flow path characterising the combustor (see also Fig. 1) is modelled as a string of consecutive straight ducts with different cross-sectional areas and/or mean radii to account for the axially varying geometry. Appropriate conservation conditions are applied across the junctions of ducts and zones of heat input. The premixers are modelled as a ring of ducts uniformly positioned circumferentially. The combustor boundary conditions applied are those of a choked inlet and outlet [22], representing approximations for the compressor outlet and turbine inlet flow respectively.

The power spectral density, $\widehat{P}(\mathbf{x}, \omega)$, is the statistical quantity measured in the experiments and defined as:

$$
\widehat{P}(\mathbf{x}, \omega)=\int_{V_{f}}\left|\widehat{G}_{1}(\mathbf{y}, \mathbf{x}, \omega)\right|^{2} \psi_{q}(\mathbf{y}, \omega) V_{c o r} \mathrm{~d}^{3} \mathbf{y}
$$

where $\psi_{q}$ is the power spectral density of the heat release fluctuations and $V_{c o r}$ is the correlation volume. In Eq. 3, the product of heat release rate spectrum $\psi_{q}(\mathbf{y}, \omega)$ and its correlation volume needs to be integrated over the flame brush. This is equivalent to the integration of the contributions from coherent sources of combustion noise with a correlation volume $V_{c o r}$ over the flame volume. For this purpose, the spectral model initially proposed by Hirsch et al. [23], modified by Liu et al. [20] and further extended to partially premixed flames has been used in this work. As suggested in previous studies [24,25], the correlation volume has been modelled as $V_{\text {cor }}=8 l_{\text {cor }}^{3}$, where $l_{c o r} \approx \delta_{T}$ is the flame brush thickness. The location of the flame and the correlation volume have been determined by means of a RANS simulation of the combustor. Finally, the sound pressure level (SPL) is found from the power spectral density as $10 \log _{10}\left(\widehat{P}(\mathbf{x}, \omega) / p_{\text {ref }}^{2}\right)$ where the reference acoustic pressure is $p_{r e f}=2 \times 10^{-5} \mathrm{~Pa}$.

\section{Turbine model}

To study the combustion noise propagation and generation within the turbine blade rows of the demonstrator aeroengine, the LINEARB code has been used $[18,19]$. The LINEARB program was developed in the early 1980's to calculate the distortion transmission 
for a high hub-to-tip ratio compressor. For the purpose of this work, a revised version of the original LINEARB code is used to solve the transmission of perturbations through aeroengine turbine stages. In this code each blade row is modelled by a control volume over which flow quantities are averaged and similar to the actuator disc theory (ADT) of Cumpsty and Marble [26] each blade row is replaced by an actuator disk. A problem with the use of actuator disks is, however, that if the volume of fluid in the model is equal to that in the machine, so that inertia effects are correctly modelled, then the spacing between the disks must be of the order of the mid-chord to mid-chord distance between adjacent blade rows. This leads to a greatly increased facility for circumferential cross flow. In order to accommodate these types of effects simultaneously, models based on semi-actuator disks were developed. Thus, the formulation and solution of a disk model can be considered in two parts. The first is the solution of the unsteady compressible equations for small disturbances in regions of uniform mean flow. The second part is concerned with the description of how such disturbances interact with the stator/rotor which is modelled by semi-actuator disks. In the control volumes between actuator disks, mean flow quantities are assumed to take known, uniform and steady values. The geometry of the turbine and the mean flow data are required for the computation. This data was obtained from numerical analysis with the in-house code Q263 and provided by Rolls-Royce.

The LINEARB code allows to investigate the propagation and generation of direct and indirect combustion noise as acoustic, entropic and vortical waves propagate and interact in the turbine blade rows and the jet pipe. LINEARB considers incoming disturbances onto the turbine from the combustor representing any of downstream propagating acoustic waves, or vorticity or entropy fluctuations. The outputs of the code are the perturbations in the jet pipe normalised on the inputs, i.e. LINEARB outputs the transfer functions which relate the pressure fluctuation at the outlet with any of the perturbations at the turbine inlet. In particular, for each circumferential mode $n$, transfer functions for the incoming pressure and entropy perturbations, $\left(\widehat{p}_{\text {jetpipe }} / \widehat{p}_{\text {in }}\right)_{n}$ and $\left(\widehat{p}_{\text {jetpipe }} / \widehat{s}_{\text {in }}\right)_{n}$, can be obtained. Perturbations at intermediate stages in the turbine can also be obtained from the computation. The turbine was considered as isolated, i.e. with a non-reflecting boundary condition at the jet pipe exit. Furthermore, since in the conditions studied here the nozzle guide vane, $\mathrm{NGV}$, at the exit of the combustor is choked, acoustic perturbations generated in the turbine do not enter the combustor.

\section{Entropy dispersion model}

Based on previous work [11-13], a model for the transport of entropy waves in real combustors has been developed. Keeping in mind that diffusion and turbulent mixing may give additional contribution to the decay of the entropy fluctuations, especially in the case of small wavelengths of the entropy perturbation (e.g. compared to a turbulent length scale) and long distances travelled by the wave [13], the model proposed here is based on the convection of entropy fluctuations along mean streamlines and therefore considers only the dispersion due to a non-uniform mean velocity profile as attenuation mechanism of the entropy perturbation averaged over the combustor cross section. Assuming that the entropy fluctuations are generated at the flame location (this assumption is reasonable for lean burn combustors [3]) and that the phase of the entropy fluctuations is the same at each location of the flame (i.e. the flame is thin), the phase shift of entropy perturbations travelling along different streamlines at the combustor exit can be described in terms of differential time delay. For a generic $k$-th streamline, the time delay $\bar{\tau}_{k}$ of a perturbation departing from the flame and reaching the exit of the combustor can be computed starting from the flow velocity along the streamline. By defining a regular array of streamlines at the combustor outlet, it is possible to decompose the flow at the combustor exit in an ensemble of many streamtubes. Considering a reasonably large number of streamlines (streamtubes) and assuming uniform properties at each cross section of the streamtube, a mean value of the entropy fluctuation at the combustor outlet (useful for low-order models) can be obtained as:

$$
\frac{\widehat{s}_{\text {outlet }}}{\widehat{s}_{\text {flame }}}=\frac{1}{\dot{M}} \sum_{k=1}^{N} \dot{m}_{k} \exp \left(-2 i \pi f \bar{\tau}_{k}\right)
$$

where $\dot{M}=\sum_{k=1}^{N} \dot{m}_{k}$ is the total mass flow rate, $\dot{m}_{k}$ is the mass flow rate associated to each streamtube and $N$ is the total number of streamtubes used for the computation.

An example of mean streamlines in the investigated combustor is shown in Fig. 2. It is important to point out that the presence of a swirling flow can cause a big dispersion of the time delay due to the complex flow field generated in the combustor. The values of the magnitude of the entropy transfer function determined through the model of Eq. 4 are shown in Fig. 3 for different numbers $N$ of streamtubes to demonstrate the convergence for high values of $N$ (all the computations presented in the following were performed using converged values of the entropy transfer function). It is remarkable that the shape of the magnitude of the entropy transfer function shown in Fig. 3 is quite similar to the one found in Refs. $[12,13]$ for fully developed channel and pipe flows.

It is also interesting to analyze the entropy transfer function in various sections of the combustor to highlight which region gives the most important contribution to the overall decay of the entropy perturbations. The different zones considered for this analysis are shown 
in Fig. 4. The transfer function of each region was computed by directly applying the model of Eq. 4 from the inlet to the outlet of each section, implicitly assuming uniform entropy fluctuations at the respective inlet planes. It is important to note that, in general, the entropy field in a given cross-section is not uniform since influenced by the convection in the upstream region and therefore the overall transfer function (from the flame to the combustor outlet) cannot simply be obtained as the product of transfer functions evaluated by applying the model of Eq. 4 to each zone independently [13]. The magnitude of the entropy transfer function corresponding to each zone is shown in Fig. 5. The highest contribution to the decay of entropy fluctuations comes from the first two sections of the combustor where the presence of the recirculation region causes a wide dispersion of the time delay, whereas the last section of the combustor gives only a marginal contribution to the overall decay (this is due to the short length of this section and the high bulk velocity which reduce the dispersion of the time delay, i.e. low He numbers as defined in [13]).

The entropy transfer function introduced in Eq. 4 has been included in the combustor model developed in LOTAN. In the following, results without and with dispersion of entropy waves will be discussed. In the first case the entropy waves are just convected with a mean time delay, computed from the bulk velocity of the combustor in each section and no attenuation due to the dispersion is considered (as if all the streamlines had the same time delay). Note that, as discussed in [13], when a non-uniform mean velocity profile is present, the mean convection velocity of the entropy spots may be different from the one resulting from the bulk velocity. When dispersion of entropy waves is considered, the model of Eq. 4 is used. This allows to include the attenuation due to the differential time delay as well as to consider a more realistic convection velocity for the average perturbation over the combustor cross section.

\section{RESULTS AND DISCUSSION}

The computation with LOTAN allows to determine the pressure and entropy fluctuation at every section of the combustor and to compute transfer functions (for example at the exit of the combustor) of the form $\widehat{p}_{\text {comb.exit }} / \widehat{q}$ and $\widehat{s}_{\text {comb.exit }} / \widehat{q}$. These can be combined with the transfer functions predicted by LINEARB for the turbine to obtain the overall transfer function $\widehat{p}_{\text {jetpipe }} / \widehat{q}$ for the prediction of the broadband combustion noise at the exit of the demonstrator engine. The measurements available from the experiment are the pressure levels at the exit of the turbine, inside the combustor and in the outer annulus (see also Fig. 1). In the following, numerical results will be compared to the experiments in terms of SPL (determined through Eq. 3) and magnitude of an overall pressure transfer function, $T F$, defined as the ratio between the pressure level in the jet pipe and the one inside the combustor, i.e. $T F=\widehat{p}_{\text {jetpipe }} / \widehat{p}_{\text {comb }}$. This can be obtained directly from the transfer functions computed by LOTAN and LINEARB.

\section{Noise prediction without entropy dispersion}

Numerical results from a computation performed without considering the dispersion of entropy waves (convection of the entropy perturbations modelled with a uniform time delay) are considered in this section. Comparisons between the experiment and numerical prediction in terms of pressure level at the location inside the combustor and in the outer annulus are first discussed to demonstrate the overall reliability of the low-order model of the combustor to capture the noise level generated by the flame. The transfer functions obtained through LOTAN for the two locations are combined with the spectral model of the flame (see Eq. 3) to predict the broadband combustion noise at the two selected locations. The direct combustion noise can be extracted by adding the downstream and upstream propagating acoustic waves and neglecting the contribution coming from the acceleration of entropy waves at the choked nozzle. The indirect noise due to the entropy wave can be determined by subtracting the direct noise from the total noise. Results for the outer annulus and the combustor are shown Figs. 6 and 7, respectively. As can be seen by comparing the results at the two locations, the difference in the pressure spectra between the combustor and outer annulus is captured reasonably well by the present low-order model. Overall, the broadband levels predicted by the numerical simulation are in reasonable agreement with the experiment. It is interesting to note that the main contribution to the sound pressure level is given by the direct noise (pressure fluctuations directly generated by the unsteady combustion) whereas the indirect component (due to the acceleration of entropy waves at the combustor outlet) is less relevant (therefore the introduction of a model for the entropy dispersion would not determine significant changes of the overall noise at these two locations). A strong combustion resonance occurs at $410 \mathrm{~Hz}$ and its harmonic at $820 \mathrm{~Hz}$ in both the outer annulus and in the combustor, generating a narrow peak that dominates the pressure spectra. This is due to self-excited narrow-band thermoacoustic oscillations of the combustion system. The frequencies of the combustion oscillations can be predicted using LOTAN provided the calculation includes a description of the feedback through which the flow perturbations modify the rate of combustion. Although very interesting for the combustor design, the investigation of the occurrence of thermoacoustic instabilities is beyond the scope of this work, where the focus is on the broadband combustion noise, and therefore the possible presence of unstable modes will not be further discussed.

Figure 8 shows the ratio between the pressure level at the exit of the turbine and inside the combustor. Comparison with the experiment shows that the magnitude of the transfer function is in general overpredicted at intermediate and high frequencies. Generally 
speaking, the noise level at the turbine exit can be decomposed into three main contributions: (i) downstream travelling pressure waves generated by the flame in the combustor, the so called direct noise (green line in Fig. 8); (ii) downstream travelling pressure wave generated by acceleration of entropy waves and its reflection in the combustor (first part of the indirect noise, yellow line in Fig. 8); (iii) the entropy perturbation generated by the flame convects to the combustor outlet and can accelerate through the turbine stages producing additional noise (second part of the indirect noise, blue symbols in Fig. 8). Investigation of the relative importance of each contribution, performed by comparing the transfer function associated to each of them, shows that the indirect noise is prevalent over the direct component and the overprediction at intermediate and high frequencies can mainly be ascribed to the noise generated through the acceleration of entropy perturbations through turbine blade rows. This implies that the magnitude of the entropy perturbations are highly over-predicted and this is expected to be caused by the absence of a model for the diffusion and dispersion of entropy waves as they convect through the combustor and turbine blade rows. In the simulations discussed here, both in LOTAN and LINEARB it is assumed that the entropy perturbations remain intact, as they move through the different components of the engine. However, in reality, shear dispersion (differential time delay) as well as turbulent mixing and diffusive phenomena can lead to high attenuation of entropy waves. This will be demonstrated in the next section where numerical results from simulations performed including the model for entropy dispersion in the combustor will be analyzed.

\section{Noise prediction with entropy dispersion}

Predictions of combustion noise by including entropy dispersion inside the combustor are discussed in the following. Comparisons with experiments in terms of magnitude of the overall pressure transfer function are shown in Fig. 9. Results show a good prediction at both low $(f<100 \mathrm{~Hz})$ and high $(f>1000 \mathrm{~Hz})$ frequencies, whereas at intermediate frequencies the magnitude of the pressure transfer function is overpredicted. It should be noted again that the present model for the transport of entropy waves considers only the differential time delay along different streamlines computed from the mean velocity field and does not include the effects of diffusion and turbulent mixing. Such effects can be very pronounced in a real combustor geometry due the presence of strong swirling flows and recirculation regions which highly enhance the mixing in the primary reacting zone. This further contributes to the decay of entropy fluctuations and the influence is expected to be very important at intermediate frequencies whereas at high frequencies the contribution of the mean flow dispersion is already sufficient to considerably attenuate the entropy perturbation. In addition to that, it should be noted that in the present model of the turbine, the entropy is simply convected without considering any effect due to the diffusion and dispersion. However, dispersion and diffusion might also play a relevant role inside the turbine further contributing to the reduction of the indirect noise. This is further demonstrated in Fig. 10 where the limiting case of entropy waves completely diffused after the first stage of the turbine (nozzle guide vane, NGV) has been considered (this simulation was performed without including the entropy dispersion model in the combustor). With such assumption the noise prediction at intermediate and high frequencies is in quite good agreement with the experiment, whereas at low frequency an underprediction of the magnitude of the pressure transfer function is observed.

This suggests that the prediction of combustion noise in real engines by low-order codes can be improved by modifying the theoretical model for the entropy convection in the combustor to include the effect of turbulent mixing and diffusion, and by developing a model for the diffusion and dispersion of entropy waves passing through the turbine blades.

\section{CONCLUSIONS}

The effect of entropy dispersion on the level of combustion noise generated in a realistic engine has been investigated using loworder acoustic network codes. A model for the decay of entropy waves inside the combustor has been proposed. This model is based on the dispersion due to a non-uniform mean velocity profile and requires only a mean velocity field, obtained through a RANS simulation of the combustor, as an input. The introduction of the entropy dispersion in the combustor model results in an overall attenuation of the noise level at the exit of the engine and in general allows us to improve the agreement with the experiments, especially at high frequencies, compared to the case where the entropy waves are simply convected without attenuation of their magnitude. This highlights the very important role of entropy wave dispersion and diffusion in determining the noise level at the exit of the engine. Some discrepancies appear at intermediate frequencies where the noise level is still overpredicted. The discrepancies between the numerical results and the experiment at intermediate frequencies may be attributed to role of turbulent mixing on the decay of entropy waves inside the combustor, not considered in the proposed model, and to the absence of a proper model for the diffusion and dispersion of entropy fluctuations in the turbine network, which would increase the attenuation of such waves. Both these areas require further investigation and model development and this should be addressed in future work. 


\section{ACKNOWLEDGMENT}

The research leading to these results has received funding from the European Community's Seventh Framework Programme (FP7/2007-2013) under Grant Agreement n. 265586 and was conducted within the IMPACT-AE (Intelligent Design Methodologies for Low Pollutant Combustors for Aero-Engines) project. The authors also would like to thank Rolls-Royce plc. for providing the numerical simulation data necessary for the low-order network modelling.

\section{REFERENCES}

[1] Smith, M., 2004. Aircraft Noise. Cambridge Aerospace Series. Cambridge University Press.

[2] Strahle, W. C., 1972. "Some results in combustion generated noise". Journal of Sound and Vibration, 23(1), pp. 113 - 125.

[3] Dowling, A. P., and Mahmoudi, Y., 2015. "Combustion noise". Proceedings of the Combustion Institute, 35(1), pp. 65 - 100.

[4] Strahle, W. C., 1978. "Combustion noise". Progress in Energy and Combustion Science, 4(3), pp. 157 - 176.

[5] Marble, F., and Candel, S., 1977. "Acoustic disturbance from gas non-uniformities convected through a nozzle". Journal of Sound and Vibration, 55(2), pp. $225-243$.

[6] Chu, B.-T., and Kovásznay, L. S. G., 1958. "Non-linear interactions in a viscous heat-conducting compressible gas". Journal of Fluid Mechanics, 3(5), pp. 494-514.

[7] Richter, C., Panek, Ł., Krause, V., and Thiele, F., 2009. Investigations Regarding the Simulation of Wall Noise Interaction and Noise Propagation in Swirled Combustion Chamber Flows. Springer Berlin Heidelberg, pp. 217-238.

[8] Poinsot, T., 2016. "Prediction and control of combustion instabilities in real engines". Proceedings of the Combustion Institute.

[9] Eckstein, J., Freitag, E., Hirsch, C., and Sattelmayer, T., 2004. "Experimental study on the role of entropy waves in low-frequency oscillations in a rql combustor". Journal of Engineering for Gas Turbines and Power, 128(2), pp. 264-270.

[10] Stow, S. R., and Dowling, A. P., 2001. "Thermoacoustic oscillations in an annular combustor". Proceedings of the ASME Turbo Expo(2001-GT-0037).

[11] Sattelmayer, T., 2003. "The interaction of entropy fluctuations with turbine blade rows; a mechanism of turbojet engine noise". Journal of Engineering for Gas Turbines and Power, 125, pp. 11-19.

[12] Morgans, A. S., Goh, C. S., and Dahan, J. A., 2013. "The dissipation and shear dispersion of entropy waves in combustor thermoacoustics". Journal of Fluid Mechanics, 733.

[13] Giusti, A., Worth, N. A., Mastorakos, E., and Dowling, A. P., 2017. "Experimental and numerical investigation into the propagation of entropy waves". AIAA Journal, 55(2), pp. 446-458.

[14] Wassmer, D., Schuermans, B., Paschereit, C. O., and Moeck, J. P., 2016. "An acoustic time-of-flight approach for unsteady temperature measurements: Characterization of entropy waves in a model gas turbine combustor". Journal of Engineering for Gas Turbines Power, 139(4).

[15] Motheau, E., Nicoud, F., and Poinsot, T., 2014. "Mixed acoustic-entropy combustion instabilities in gas turbines". Journal of Fluid Mechanics, 749, pp. 542-576.

[16] Stow, S. R., and Dowling, A. P., 2009. "A time-domain network model for nonlinear thermoacoustic oscillations". Journal of Engineering for Gas Turbines and Power, 131(3).

[17] Dowling, A. P., and Stow, S. R., 2003. "Acoustic analysis of gas turbine combustors". Journal of Propulsion and Power, 19(5).

[18] Auer, L. H., and Heasley, J. N., 1976. "An alternative formulation of the complete linearization method for the solution of non-lte transfer problems". The Astrophysical Journal, 205.

[19] Knobloch, K., Guerin, S., Holewa, A., Mahmoudi-Larimi, Y., Hynes, T., and Bake, F., 2016. "Noise transmission characteristics of a high pressure turbine stage". Proceedings of the 22nd AIAA/CEAS Aeroacoustics Conference(AIAA 2016-3001).

[20] Liu, Y., Dowling, A. P., Swaminathan, N., Morvant, R., Macquisten, M. A., and Caracciolo, L. F., 2014. "Prediction of combustion noise for an aeroengine combustor". Journal of Propulsion and Power, 30(1), pp. $114-122$.

[21] Dowling, A. P., Swaminathan, N., Hynes, T., and Mahmoudi-Larimi, Y., 2015. Deliverable D4.3: Report on analytical prediction tool and comparison with full-scale data. RECORD project. Tech. rep., University of Cambridge.

[22] Stow, S. R., Dowling, A. P., and Hynes, T. P., 2002. "Reflection of circumferential modes in a choked nozzle". Journal of Fluid Mechanics, 467, pp. 215-239.

[23] Hirsch, C., Wsle, J., Winkler, A., and Sattelmayer, T., 2007. "A spectral model for the sound pressure from turbulent premixed combustion". Proceedings of the Combustion Institute, 31(1), pp. 1435 - 1441.

[24] Swaminathan, N., Xu, G., Dowling, A. P., and Balachandran, R., 2011. "Heat release rate correlation and combustion noise in premixed flames". Journal of Fluid Mechanics, 681, pp. 80-115. 
[25] Swaminathan, N., Balachandran, R., Xu, G., and Dowling, A., 2011. "On the correlation of heat release rate in turbulent premixed flames". Proceedings of the Combustion Institute, 33(1), pp. 1533 - 1541.

[26] Cumpsty, N. A., and Marble, F. E., 1977. "The interaction of entropy fluctuations with turbine blade rows; a mechanism of turbojet engine noise". Proceedings of the Royal Society of London A: Mathematical, Physical and Engineering Sciences, 357(1690), pp. 323-344. 


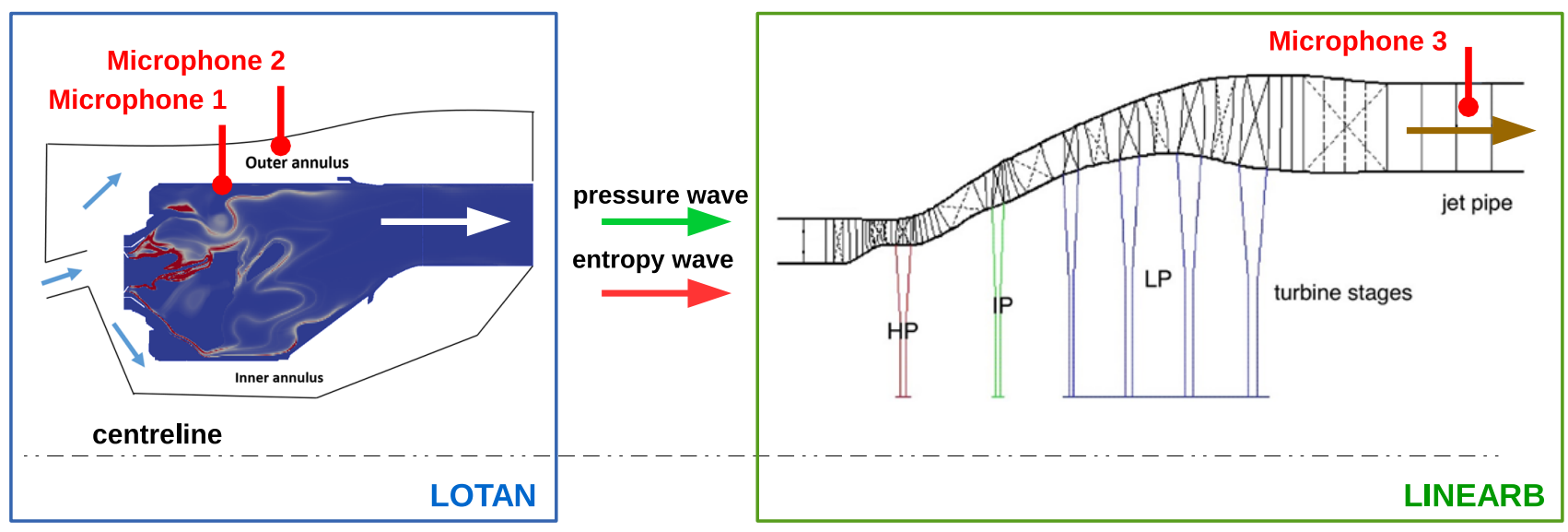

FIGURE 1. Schematic of the Rolls-Royce ANTLE combustor.

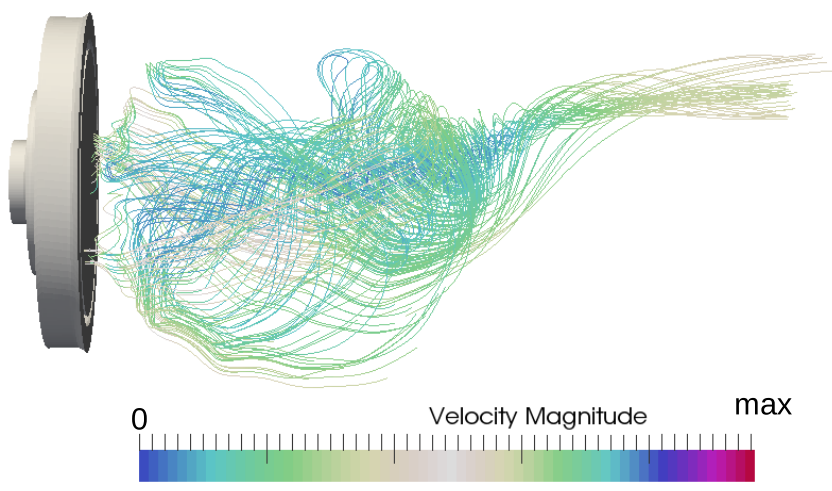

FIGURE 2. Example of streamlines obtained through a RANS simulation of the demonstrator engine combustor.

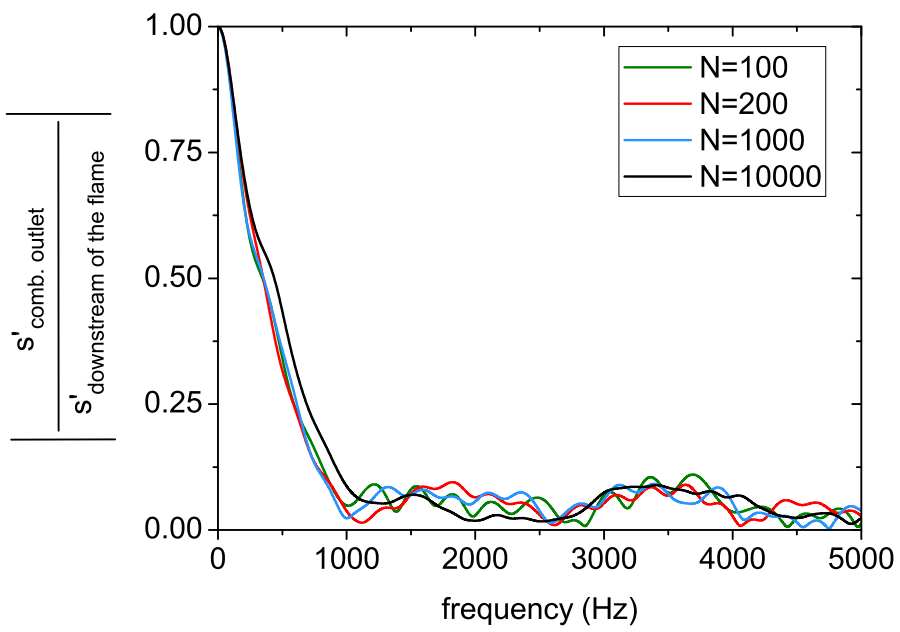

FIGURE 3. Magnitude of the entropy transfer function evaluated with different number of streamlines. 


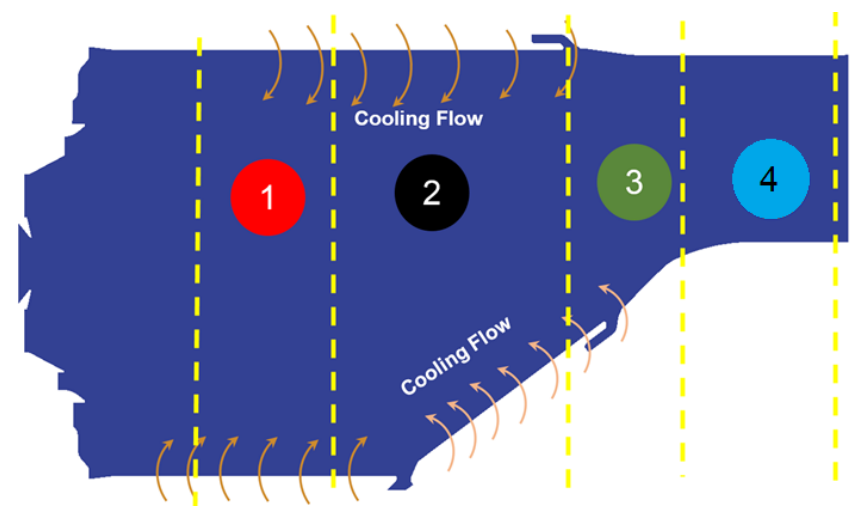

FIGURE 4. Different regions in the combustor for the evaluation of the contribution of each zone to the attenuation of entropy waves.

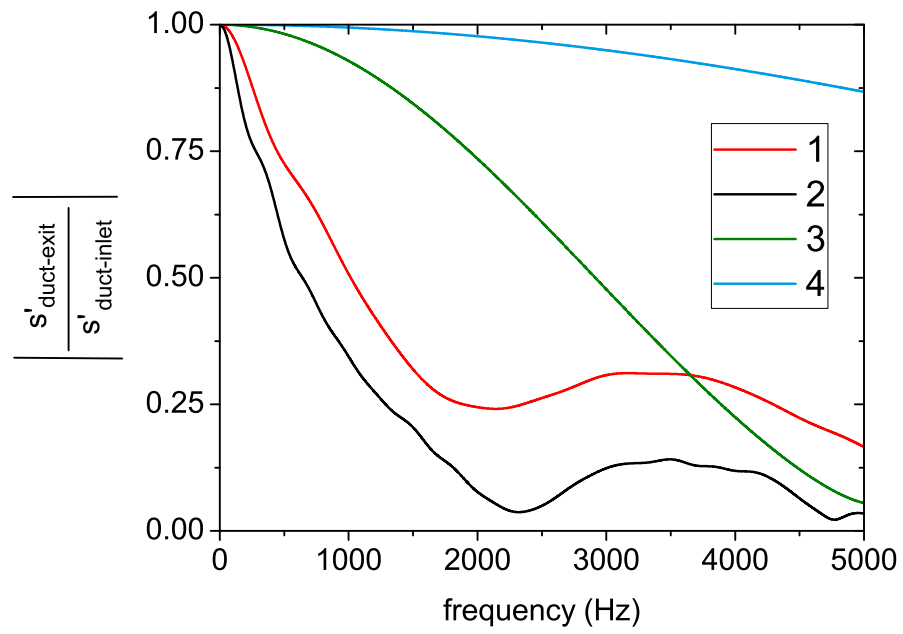

FIGURE 5. Magnitude of the entropy transfer function for the different regions of Fig. 4.

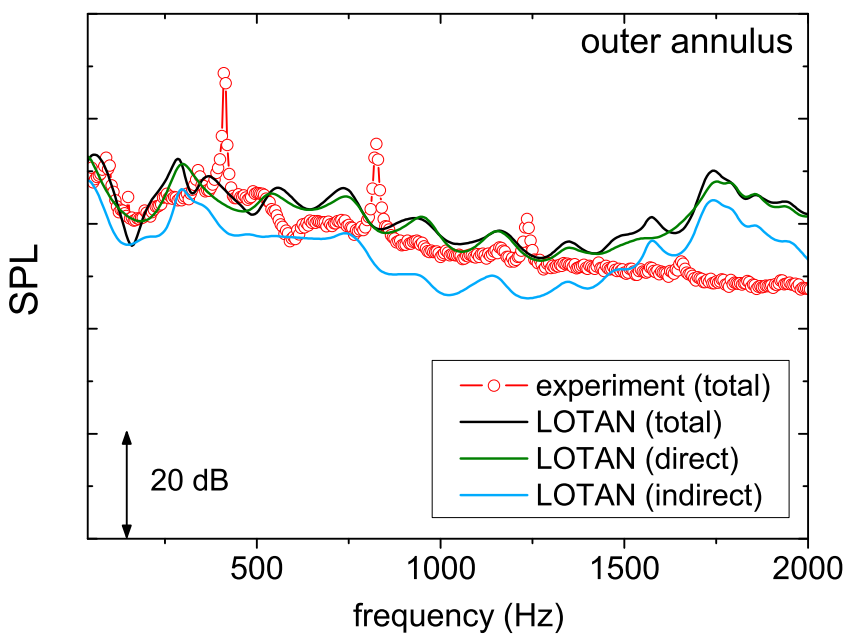

FIGURE 6. Comparison between predicted and measured noise spectra in the outer annulus of the demonstrator engine (see Microphone 2 in Fig. 1). 


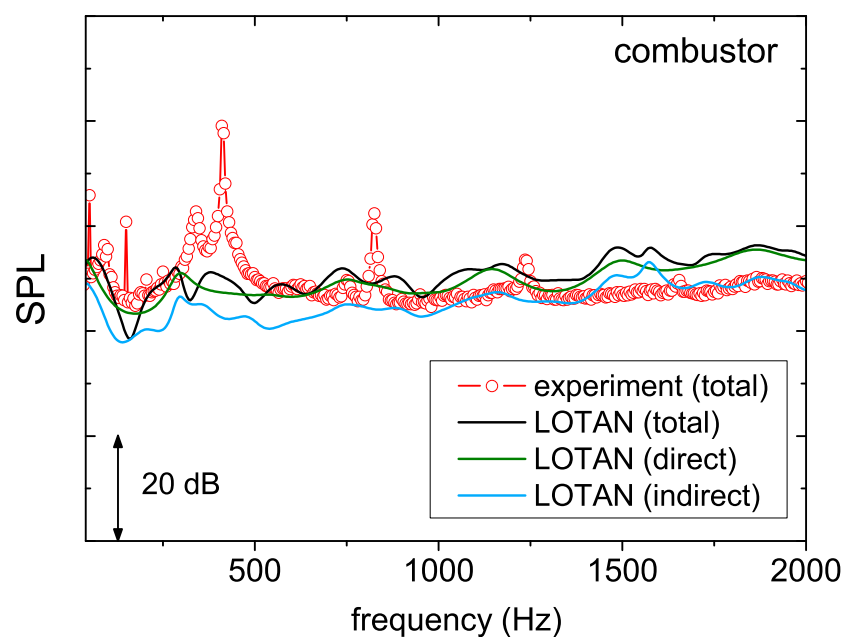

FIGURE 7. Comparison between predicted and measured noise spectra in the combustor of the demonstrator engine (see Microphone 1 in Fig. 1).

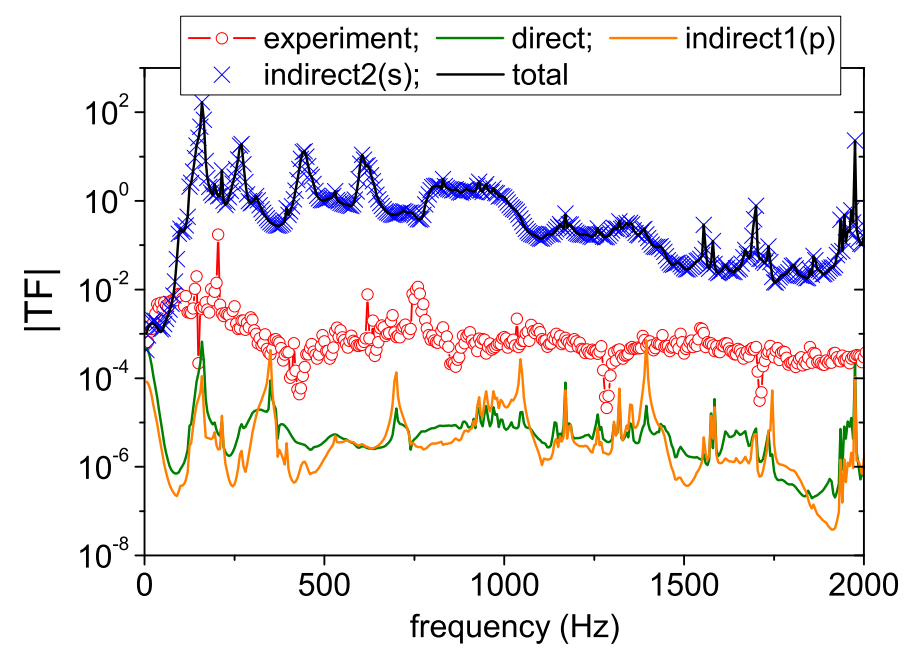

FIGURE 8. Magnitude of the pressure transfer function predicted without entropy dispersion model in the combustor; the contribution of direct and indirect noise is also included. 


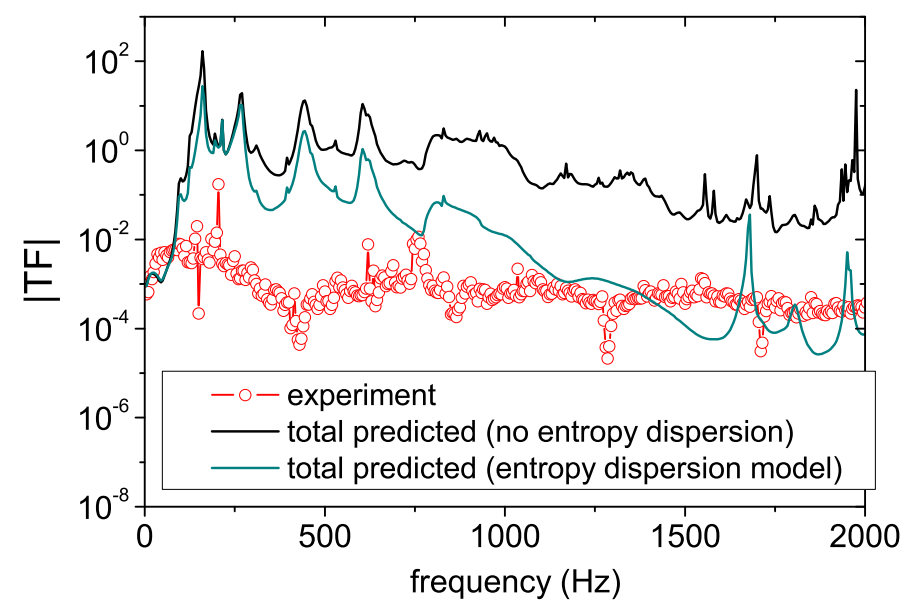

FIGURE 9. Magnitude of the pressure transfer function predicted by including the entropy dispersion inside the combustor (model of Eq. 4).

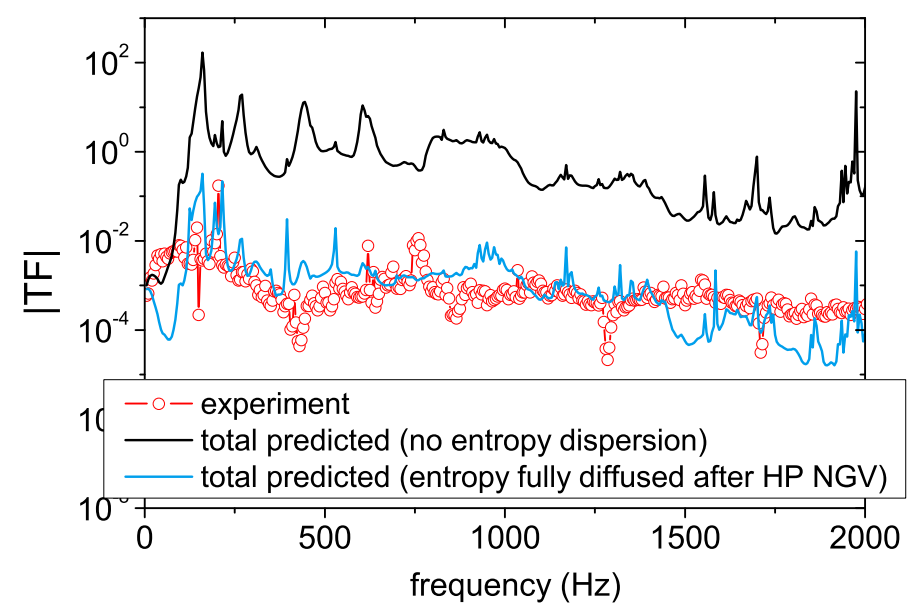

FIGURE 10. Magnitude of the pressure transfer function for the limiting case of entropy completely diffused after the first stage of the turbine. 